\title{
Fading procedure effects on a visual pattern discrimination reversal in the albino rat
}

\author{
S. ARONSOHN, O. CASTILLO, and T. PINTO-HAMUY \\ Department of Physiology and Biophysics, University of Chile, Santiago, Chile
}

\begin{abstract}
Two groups of albino rats were trained on a simultaneous pattern discrimination task, one with a classical procedure in which the discriminanda remained invariant throughout training, and the other with a fading procedure in which the final task was approached through a series of graduated steps. In a subsequent reversal stage, during which the initial positive stimulus became negative and vice versa, each group was subdivided so that half of the subjects in each original group received reversal training with the same procedure as during acquisition and the other half with the other method. Fading procedures yielded a more proficient performance on every occasion, independently of experimental stage or previous learning history. Results are analyzed in terms of favorable conditions offered by the procedure itself and of beneficial in. fluences exerted over subsequent learning.
\end{abstract}

Classical procedures used to train a discrimination learning task consist of the simultaneous or successive presentation of two or more stimuli whose characteristics remain invariant throughout training. While occasional exceptions can be found (e.g., Schlosberg \& Solomon, 1943), most studies maintain the discriminative stimuli at constant values throughout training.

Terrace (1963) introduced a technique referred to as "fading." This procedure is designed to modify stimulus control of a response by presenting initial stimuli that differ maximally, and which, through a series of graduated steps, from easy to difficult, eventually come to differ on the dimension required to control behavior. This procedure permits errorless or near errorless learning. Terrace designed this method for investigating behavior in the laboratory. It has been applied to basic research problems relevant to learning mechanisms (Pinto-Hamuy, Espinoza \& Aronsohn, 1972; Schusterman, 1966; Stettner \& Matyniak, 1969; Terrace, 1963), to the programming of instructional material (Holland, 1960; Skinner, 1958), and to the therapeutic modification of deviant or deficient aspects of behavior (Barlow \& Agras, 1973; Striefel \& Wetherby, 1973).

Sidman and Stoddard (1967) obtained partial success applying a fading procedure with retardates who had not been able to learn a form discrimination with the classical procedure. Touchette (1968) reported that "retarded children who show no signs of learning a discrimination by trial and error, can be taught by a program of graduated stimulus changes."

This paper was originally received during the tenure of the previous editor, Abram Amsel. The research was supported by NIH Grant 1-R01-NS-12470-01.
Besides reporting the beneficial effects of the fading procedure per se, this author also mentions that a previous history of error performance interferes with the acquisition of a discrimination with the fading method. Reversal learning problems appear to be a suitable means to study this interference effect. Several authors (D'Amato \& Jagoda, 1961; Spence, 1945) have shown that reversal is much more difficult than the original discrimination. However, there is some evidence that fading procedures might overcome the difficulty of a reversal task. Schusterman (1966), with two sea lions who had initially been trained with the fading method, obtained near errorless reversal in a form discrimination by means of fading. Although the sample was small, the results were promising. Similarly, Gollin and Savoy (1968) trained children in a form discrimination reversal task by applying fading procedures. Compared to controls, the greater percentage of subjects trained with this procedure did not commit errors in reversal.

The latter experiments employed fading in both stages of learning. It is, therefore, not clear whether the favorable effects of stimulus graduation in reversal are due to the application of this technique at this stage or due to the influence exerted by a prior history of errorless learning. No attempt so far has been made to assess independently the influence of each procedure on subsequent training. Furthermore, it appears convenient to extend the experimentation to other species. The present study explores the rat's performance in reversal discrimination learning, applying a fading procedure. Our interest focused on determining the separate influence of the technique on each of the experimental stages. After training two groups of rats in a simultaneous pattern discrimination with classical and fading techniques, respectively, each group was 
subdivided during the reversal stage, so that half of the rats in each group received reversal training with the same procedure as during acquisition, and the other half with the other method.

\section{METHOD}

\section{Subjects and Apparatus}

The subjects were 20 experimentally naive male albino rats, aged 3 months, whose ad-lib weights ranged from 250 to $310 \mathrm{~g}$. They were put on a deprivation schedule until they reached $85 \%$ of their initial weights. The rats were housed in individual cages, where they received about $15 \mathrm{~g}$ of food daily after each experimental session, with free access to water.

The apparatus was a modified Lashley jumping stand which consisted essentially of a wooden roofless box, $107 \times 46 \times 50 \mathrm{~cm}$, with a central $V$-shaped platform $15 \mathrm{~cm}$ above the floor of the box. The arms of the $\mathrm{V}$ were facing two wooden panels placed at an angle of $130^{\circ}$ and with $14 \times 14 \mathrm{~cm}$ openings. These led to bilateral compartments where reinforcement was available. Metal stimulus-bearing plates could be inserted from above into each opening, in front of which landing platforms were disposed. For further details see Mackintosh (1962).

Illumination was provided by two $10-\mathrm{W}$ bulbs, inserted inside metal tubes in both upper corners of the rear wall. Each light beam focused onto the respective stimulus plate. The metal tube had a lateral cut through which plastic filters of different widths could be introduced whenever the light graduation procedure was in effect. Filter 1 provided no illumination, while Filters 2, 3,4 , and 5 provided an illumination of $1,2,2.5$, and $3.5 \mathrm{fc}$, respectively. The discriminanda, consisting of an upright and an inverted white triangle on a black background, were placed simultaneously on the stimulus-bearing plates. Since there seems to be no preference for either stimulus (Munn, 1950), the upright triangle was positive for all subjects.

\section{Procedure}

All rats were given 20 daily trials until they reached a final criterion performance of at least $80 \%$ correct responses on 2 consecutive days, in each experimental stage. Forty-five milligram pellets were used as reinforcement.

The instrumental response was shaped before discrimination training began.

A trial began when the animal was placed on the starting platform. The animal's task was to jump onto one of the landing platforms. A correct response was followed by the experimenter's lifting of the corresponding stimulus plate, thus permitting the animal access to reinforcement. In case of an incorrect choice, no reinforcement was delivered. Subsequently, the rat was returned to a cage where it remained during the 2 -min intertrial interval. Either side was designated as positive, according to a chance series, with the restriction that no one side was correct for more than two consecutive trials, and with each side being reinforced on $50 \%$ of the daily trials.

During the first two sessions of each experimental stage, a correction procedure was used. When the animal committed an error, it was replaced on the starting platform. After the third incorrect jump, it was manually guided towards the opposite side. This procedure guaranteed that the subject received reinforcement on every trial of the initial sessions (Mackintosh, 1965).

Initial discrimination training. Control animals were trained to discriminate between the two triangles of the same size throughout. (Since no provision was taken to equate total luminous flux for the triangles, all references to size modifications should be understood as size-brightness variations.)

Experimental subjects were faced with a 17-step sequential procedure in which both size and illumination of the negative stimulus were modified. Advancement from one step to the next required the performing of four consecutive correct choices.
Figure 1 presents individual area and size proportion for each pair of triangles, along with a graph of some representative steps of the modification of $S-$. The present sequence was constructed on an empirical basis from the results obtained in a previous pilot experiment (unpublished).

Differential training started for the experimental subjects when Filter 1 was placed on either light tube so that only the positive stimulus plate was illuminated. During the first five steps, illumination and size of the negative stimulus were simultaneously and gradually modified.

The animal was initially faced with the full-sized, fully illuminated, upright triangle vs. a solid black stimulus (no light focused) On attaining four consecutive correct responses, the next step was the presentation of the smallest triangle of the series, while Filter 2 replaced Filter 1 , thus increasing the illumination of the negative stimulus. This procedure was continued throughout the series until criterion was reached on the final discrimination (see Figure 1).

Reversal discrimination training. After initial training was terminated, reversal started. Groups initially labeled as $\mathbf{F}$ (fading) and $\mathrm{C}$ (classical), were subdivided into two further groups each, so that half of the animals initially trained with each procedure were reversed with the same technique and the other half with the other technique.

The groups were designated with initials according to the procedures employed on initial and reversal stages (FF, FC, CC, CF, $\mathrm{n}=5$ in all cases). Thus, Group FC was initially trained with the fading procedure and reversed with the classical method.

The same procedure as in the initial training was followed except that step A (Figure 1) was excluded.

\section{RESULTS}

During initial training, there was a clear superiority for the group trained with the fading procedure as

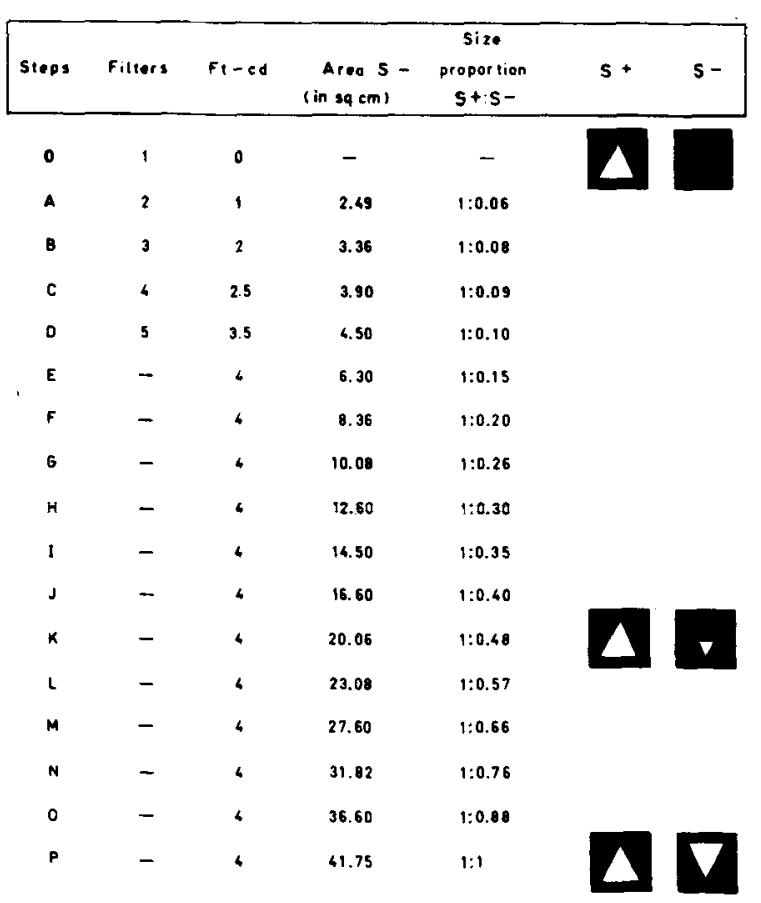

Figure 1. Step sequence of the fading procedure. Data presented in each column correspond to light projected in foot-candles for each filter, S - area (in $\mathrm{cm}^{2}$ ), and the corresponding $\mathbf{S}+: \mathbf{S}-$ size proportion. On the right, representative samples of discriminanda. 
compared to controls. Animals in Group $\mathrm{C}$ required $56 \%$ more trials to reach criterion than subjects in Group F; the group means were 152.3 and 97.7, respectively. Statistical analysis proved this difference to be highly significant $[\mathrm{t}(18)=8.719, \mathrm{p}<.005]$. The comparison of error scores showed that animals of Group C committed significantly more errors than those of Group F, $[\mathrm{t}(18)=10.868, \mathrm{p}<.001]$; the respective group means were 57.9 vs. 11.6.

The learning curves of the subjects of each group during both stages is presented in Figure 2. Note that the subjects of Groups FF and FC almost never dropped below $70 \%$ of correct responses. The subjects of Groups $C C$ and $C F$, on the other hand, reveal irregular acquisition functions, with errors scattered along the sessions; efficiency was reached late, shortly before attaining criterion. It is worth noting the similarity between curves representing the application of fading, independent of the experimental stage to which they belong (e.g., compare reversal curves of Groups FF and CF).

The mean total number of trials and errors for each group during initial and reversal stages are presented graphically in Figure 3.

Analysis of reversal data was performed for trials to criterion and errors independently by means of a 2 by 2 analysis of variance to test for the influence exerted by initial and reversal training procedures, respectively. Both experimental variables, that is, initial and reversal training procedures, had significant effects upon reversal trials to criterion $[\mathrm{F}(1,16)$ $=16.4, \mathrm{p}<.01$, and $\mathrm{F}(1,16)=57.7, \mathrm{p}<.01$, respectively]. There also was a significant interaction between training and reversal procedures $[F(1,16)=11.8, p<.01]$, suggesting that the initial training procedure effect was correlated with the reversal training procedure effect. Upon closer examination of the interaction, it is apparent that the initial training procedure effect is not significant for subjects reversed with the fading procedure (CF and FF), while the effect is pronounced for those reversed with the classical procedure (FC and $\mathrm{CC}$ ), as shown by the application of the $q$ statistic $[q(8,16)=-155$, $\mathrm{p}<.01$; Dixon \& Massey, 1957]. Conversely, the reversal training procedure effect is not significant for subjects initially trained with the fading procedure (FF and $\mathrm{FC}$ ) as it is for those initially trained with the classical procedure (CF and $C C$ ) $[q(8,16)$ $=-224, \mathrm{p}<.05]$.

Analysis of the error scores followed the same trend as trials to criterion. Again, both initial and reversal training procedures had significant effects upon reversal-stage error scores $[F(1,16)=29.5$, $\mathrm{p}<.01$, and $\mathrm{F}(1,16)=16.1, \mathrm{p}<.01$, respectively]. The interaction between the two variables was also significant $[F(1,16)=169.9, p<.01]$. Initial training procedure had no effect upon groups reversed with the fading procedure (CF and FF), while the

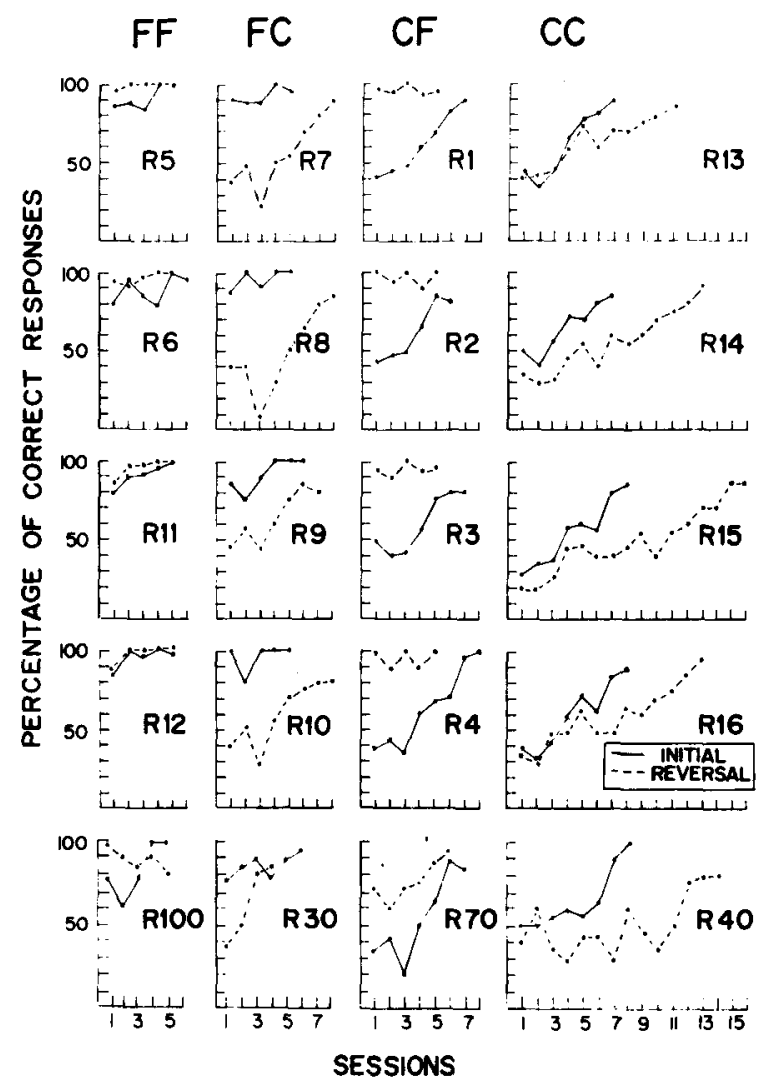

Figure 2. Initial and reversal learning curves for individual subjects of each group as a function of percentage of correct responses along sessions.

effect was significant for groups reversed with the classical procedure (FC and $\mathrm{CC}$ ) $[q(8,16)=-81$, $\mathrm{p}<.05$ ]. Reversal training procedure, on the other hand, had a significant effect both upon groups initially trained with the fading procedure (FF and

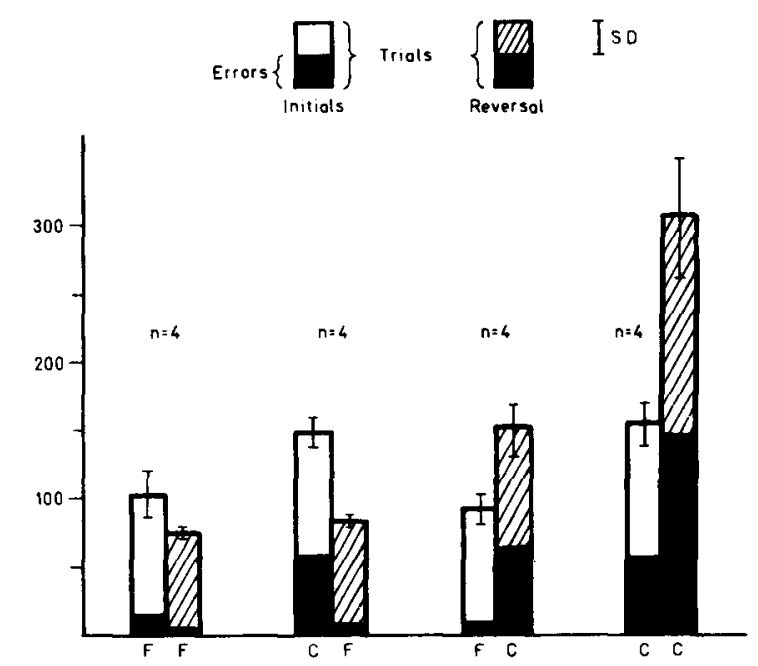

Figure 3. Mean trial and error scores during initial and reversal training for each group. Left-hand bars of each cluster indicate acquisition; right-hand bars indicate reversal. Total area of column indicates trials; shadowed area indicates errors. 
FC) $[q(8,16)=-60, p<.05]$ and upon groups initially trained with the classical procedure (CF and CC) $[\mathrm{q}(8,16)=-138, \mathrm{p}<.05]$.

\section{DISCUSSION}

As compared to the classical method, the fading procedure reduces the number of errors and number of trials required to master the reversal of a form discrimination. The successful results obtained with the albino rat in our experiment lend further generality to the results obtained with sea lions (Schusterman, 1966) and retarded children (Gollin \& Savoy, 1968). The beneficial effects of applying a fading procedure during acquisition of a discrimination confirms the results already shown by Terrace (1963) and others.

The application of the fading procedure yielded satisfactory results whenever employed, independently of experimental stage or prior training. The fact that the initial and reversal stages of Group FF were equally effective and the fact that this group did not perform differently during reversal from Group CF suggests that the fading procedure may serve to overcome a previous history of error performance. However, the most striking influence of the fading procedure upon subsequent learning appears in comparing the performance of Groups FC and CC in reversal. The impressive fact is how a steady and errorless learning process may serve to improve future learning where no provisions are made to reduce errors or speed up the process. Thus, we may conclude that the fading technique not only proves to be an efficient training procedure in itself, but also influences subsequent patterns of learning behavior.

A possible way to visualize the relevant factors that might be responsible for the easier way in which rats trained with the fading procedure reach reversal learning is depicted in Figure 4. This figure shows the final step of the discriminanda of initial training and Step A of reversal for each group. As may be seen, for both groups reversed with the fading procedure, the transition from initial to reversal stage represents a change as to the most conspicuous dimension which serves to differentiate both stimuli. For these groups, initial training ends with both stimuli differing on the pattern dimension, while reversal starts with a more clear-cut difference on the size dimension. Conversely, for subjects trained with the classical

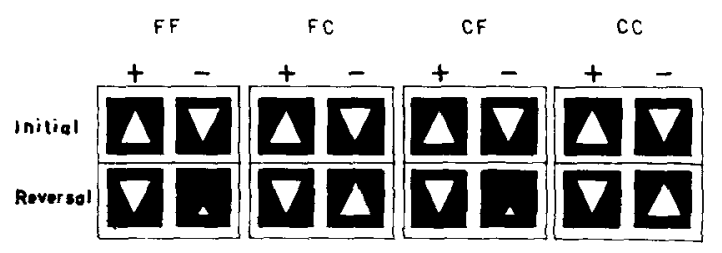

Figure 4. (Top) Final step of initial training. (Below) First step of reversal for each group. procedure, the transition from the initial to the reversal stage conveys a change of the stimulus value on the pattern dimension, exclusively.

Groups reversed with the classical procedure, on the other hand, performed as expected and in accordance with results reported in the literature showing that reversal is a more difficult task than acquisition (D'Amato \& Jagoda, 1961; Spence, 1945). Fading, on the other hand, is initiated by making use of a cue that could be thought of as more conspicuous, or having a higher ecological value or rank in the perceptual hierarchy (Jones, 1964). As training proceeds, the animal learns to respond to a different cue, transfering its initial preference of one dimension (size) to a different one (pattern). Once the criterion is met, "reversal training" starts. For the fading groups, animals are confronted with a situation which differs completely from where "initial training" ended (see Figure 4). The size cue is reintroduced and the configuration of cues is such that the problem may be solved in terms of its "preference" for this specific dimension. The fact that this stage is one of reversal is only accidental to the subject, for one could assume that the most economic way of mastering the discrimination is in terms of the "preferred" cue (size) rather than in terms of the "reversed" cue (pattern). This way, the animal is confronted with the so-called reversal problem, "as if" it were an entirely new problem, while the experimenter is handling the data "as if" it corresponded to reversal learning.

However, the subject's "preference" for a specific dimension does not guarantee that he will respond on that basis, unless the discriminative stimuli on this dimension are different enough to permit a prompt discriminative response on this basis. Lawrence (1952) stressed the importance of this fact, stating that "the most efficient way ... of setting up a discrimination is to devote part of the training trials to an easier discrimination on the same continuum of stimuli." Shepp and Zeaman (1966) further add, "Rate of discrimination learning will be directly related to physical differences between discriminanda." This way, the association of an overt response with a specific stimulus dimension is favored in the fading procedure by the initial presentation of an easy discrimination in terms of stimuli which differ maximally between them.

Our results may be compared to those obtained by North (1958), who, although not using fading, found that a group trained initially on an easy problem and then on a difficult one, took fewer trials to learn both problems than a group trained only on the difficult problem. We might say that it would be rather difficult to account for our results merely on the basis of $S-R$ connections, as "the amount learned about a given cue in a given number of trials (rate of learning that cue) depends not only on such 
static variables as stimulus-response-reinforcement contiguity, but also on the subject's prior experience with that and other cues"' (Sutherland, 1971). D'Amato and Jagoda (1961) would tend to explain these results in terms of FC subjects finding $\mathrm{S}-$ less aversive than $\mathrm{CC}$ subjects, due to the fact that the former had responded less to $\mathrm{S}$ - during the initial phase and had been less nonreinforced in the presence of this stimulus. This type of theory would predict a faster reversal for Groups FC, for these subjects would be more likely to select the former $\mathrm{S}$ - than would the subjects of Group CC, for whom the initial $\mathrm{S}-$ would be greatly aversive. This explanation however, is not consistent with the proficient results of Group CF. The latter, having a similar history of nonreinforced $\mathrm{S}-$ approaches, attained a reversal performance as efficient as that of Group FF. Therefore, any adequate interpretation of our results would necessarily have to include the assumption of additional processes appropriate to reversal learning besides the mere acquisition of approach and avoidance responses to the positive and negative stimuli. In this sense, selective attention theory seems quite adequate, since "if successful performance on a discrimination problem depends upon both learning to switch-in the relevant analyzer and learning to attach appropriate responses to the outputs of the analyzer" and "an animal is trained to switch-in a given analyzer by learning to make one set of responses to stimuli varying along the dimension in question, then its learning of a subsequent problem involving the same relevant dimension but different responses should be facilitated" (Sutherland, 1971).

Compared to $\mathrm{CC}$ subjects, $\mathrm{FC}$ subjects are favored because the initial fading procedure has probably determined a strengthening of the relevant analyzer at the outset of reversal and the subjects have only to modify their overt responses.

In spite of the advantage of the fading procedure in all instances, it should be stressed that these are a function of the design of an appropriate series. The sequence of the stimulus graduations employed is what ultimately determines the results obtained with this technique.

\section{REFERENCES}

Barlow, D. H., \& AGras, W. S. Fading to increase heterosexual responsiveness in homosexuals. Journal of Applied Behavior Analysis, 1973, 6. 355-366.

D'Amato, M. R., \& JAGoda, H. Analysis of the role of overlearning in discrimination reversal. Journal of Experimental Psychology, 1961, 61, 45-60.
Dixon, W. J., \& MASSEY, JR., F. J. Introduction to statistical analysis. Tokyo: Kogakusha, 1957.

Gollin, E. S., \& SAvoy, P. Fading procedures and conditional discrimination in children. Journal of the Experimental Analysis of Behavior, 1968, 11, 443-451.

Holland, J. G. Teaching machines: An application of principles from the laboratory. Journal of the Experimental Analysis of Behavior, 1960, 3, 275-287.

JoNEs, L. V. Distinctiveness of color, form and position cues for pigeons. Journal of Comparative and Physiological Psychology. 1964, 47, 253-257.

LAWRENCE, D. H. The transfer of a discrimination along a continuum. Journal of Comparative and Physiological Psychology, 1952, 45, 511-516.

Mackintosh. N. J. The effects of overtraining on a reversal and a nonreversal shift. Journal of Comparative and Physiological Psychology, 1962, 55, 559-589.

Mackintosh, N. J. Selective attention in animal discrimination learning. Psychological Bulletin, 1965, 42, 124-150.

Munn, N. L. Psychological research on the rat. Massachusetts: Houghton-Mifflin, 1950.

North, A. J., Maller, O., \& Hughes, C. Conditional discrimination and stimulus patterning. Journal of Comparative and Physiological Psychology, 1958, 51, 711-715.

Pinto-Hamuy, T., Espinoza, V. B., \& Aronsohn, S. Discriminación visual en ratas utilizando la técnica de "fading." Simposium Latinoamericano de Psicobiologia del Aprendizaje, Santiago-Chile. Santiago: Central de Publicaciones de la Facultad de Medicina, 1972. Pp. 105-109.

Schlosberg, H., \& Solomon, R. L. Latency of response in a choice discrimination. Joumal of Experimental Psychology, 1943, 33, 22-39.

Schusteraman, R. Serial discrimination reversal learning with and without errors by the California sea-lion. Joumal of the Experimental A nalysis of Behavior, 1966, 9, 593-600.

Shepp, B., \& Zeaman, D. Discrimination learning of size and brightness by retardates. Journal of Comparative and Physiological Psychology. 1966, 62, 55-59.

Sidman, M., \& Stodnard, L. T. The effectiveness of fading in programming a simultaneous form discrimination for retarded children. Journal of the Experimental Analysis of Behavior. 1967, 10. 3-15.

Skinner, B. F. Teaching machines. Science, 1958, 128, 969-977.

SPENCE, K. W. An experimental test of the continuity and non-continuity theories of discrimination learning. Journal of Experimental Psychology, 1945, 35, 253-266.

Stettner, L. J., \& Matyniak, K. A. The use of stimulus fading in assessing behavioral deficits produced by brain damage. Physiology and Behavior, 1969. 4, 859-861.

STRIEFEL, S., \& WETHERBY, B. Instruction-following behavior of a retarded child and its controlling stimuli. Journal of Applied Behavior Analysis, 1973, 6, 663-670.

Sutherland, N. S., \& Mackintosh, N. J. Mechanisms of animal discrimination learning. London: Academic Press, 1971.

Terrace, H. S. Discrimination learning with and without "errors." Journal of the Experimental Analysis of Behavior, $1963,6,1-27$.

Touchette, P. E. The effects of a graduated stimulus change on the acquisition of a simple discrimination in severely retarded boys. Journal of the Experimental Analysis of Behavior, 1968, 11, 39.48.

(Received for publication October 2, 1975; revision accepted July 28,1977 .) 